\title{
Mapping the musculoskeletal system one cell at a time
}

\author{
${ }^{1}$ Mathew Baldwin, ${ }^{1}$ Adam Cribbs, ${ }^{2,3}$ Farshid Guilak \& ${ }^{1}$ Sarah Snelling ${ }^{\dagger}$ \\ †e-mail: sarah.snelling@ndorms.ox.ac.uk
}

The Human Cell Atlas aims to map tissues and organs at single cell resolution during development, maturation, and pathology. The high burden of musculoskeletal disorders means such maps are critical to delivering therapeutic approaches. Here we present the musculoskeletal HCA network as a community for fostering collaboration and shared expertise.

\section{Main text}

The approximately 37 trillion cells in a human body are hugely diverse in form and function. The phenotype of each of these cells is defined by their unique epigenomic, transcriptomic and proteomic signatures. However, the individual cells of our bodies do not work in isolation but instead interact with their extracellular matrix and neighbouring cells to form increasingly complex tissues, organs and systems. Mapping the cellular composition of tissues and organs, as well as their spatial organisation, will therefore be vital to unravel how the cellular systems of the human body are maintained in health and disrupted in disease.

Over the past 150 years, biologists have attempted to classify cell types on the basis of their morphology, function and molecular expression. Although gradual technological improvements have underpinned each incremental advance in cellular indexing, our current understanding of cell types and cell states remains incomplete. Breakthroughs over the past decade in the genomic, epigenomic, transcriptomic and proteomic profiling of single cells have now made it possible to map cellular biology with more detail than ever before, paving the way for the Human Cell Atlas (HCA) project.

\section{The Human Cell Atlas}

The generation of high-quality reference maps for all human cells one of the missing, but achievable, links in understanding human development and health. The international HCA project aims to align biological, clinical and computational expertise to deliver a molecular profile of tissues and organs at single-cell resolution. The project will catalogue all the cellular phenotypes in the human body, identify their spatial location and measure phenotypes across different stages of differentiation and cell states. Importantly, the international and open-source nature of the HCA embodies the 
community's desire to build an atlas that is representative of global populations ${ }^{1}$, thus ensuring the equitable distribution of a new era of precision and regenerative medicine.

The HCA is organised into biological networks that each cover different tissues and are at different stages of development within a three-phase evolutionary model of atlas delivery. The first phase involves uniform massively parallel sequencing using single-cell and/or single-nuclear RNAsequencing to aid decision-making for an initial roadmap. This phase is termed the 'sky dive'. The second phase is a deeper analysis with specialised lower throughput methods (such as full-length single-cell RNA-sequencing) to provide detailed maps for a small number of individuals and cells. This phase is referred to as 'deep dive'. The third and final phase involves exploring patient-topatient variation to encompass age, sex, ethnicity and geographical variation to deliver a broad, representative atlas.

Although the HCA focuses predominantly on transcriptomic approaches, the integration of epigenomic and proteomic data and spatial imaging with transcriptomic data is being deployed at all phases. Building an atlas of healthy tissues is the initial priority, as this will provide a 'Rosetta stone' reference against which disease-associated changes can be deciphered.

\section{The Musculoskeletal HCA Network}

Musculoskeletal disease is the leading global cause of disability ${ }^{2}$. Worldwide, $20-33 \%$ of people live with painful and debilitating musculoskeletal diseases that affect a diverse range of functionally distinct tissues including tendon, cartilage, ligament, capsule, synovium, muscle and bone ${ }^{3,4}$. Given the ubiquity of musculoskeletal tissue, which comprises at least one-third of the weight of a human body, it is perhaps unsurprising that disease and traumatic injury affects the musculoskeletal system at all life stages, from development through to maturation and ageing ${ }^{5}$ Mapping the musculoskeletal system therefore presents an exciting challenge for the research community owing to the diversity of tissues and joints involved and the high burden of musculoskeletal disease.

Single-cell sequencing technology has traditionally been applied to tissues that are both easily accessible and amenable to analysis. Consequently, the generation of in-depth, high resolution detailing the cellular composition of lung and liver have provided exciting insights into the power of the $\mathrm{HCA}^{7,8}$. However, many musculoskeletal tissues, including tendon, ligament and cartilage, have low-cellularity and/or dense, fibrous extracellular matrices, which present new challenges for the generation of high-quality single-cell data. These challenges are further compounded by the scarce 
availability of healthy tissue samples and their small volume. The musculoskeletal HCA network, a recent addition to the HCA project, aims to circumvent such operational challenges by bringing together and aligning clinicians and researchers who are interested in similar biological topics

Ultimately, the aim of the musculoskeletal HCA network is to enable the efficient delivery of a representative omics atlas of the human musculoskeletal system (Figure 1). An atlas of healthy tissue will be complemented with disease-specific atlases representing the breadth of musculoskeletal conditions. These atlases will encompass the whole human lifespan and a variety of ethnicities, providing unprecedented insights into the molecular mechanisms underlying health and disease and helping researchers to identify new targets for the treatment, diagnosis and monitoring of musculoskeletal diseases.

To achieve this aim, we, as coordinators of the musculoskeletal HCA network, will initially work on the sky dive and deep dive phases across all musculoskeletal tissues. Methods and experience with patient recruitment, tissue collection, sample storage, annotation of tissue against anatomical landmarks and the development of standards on minimum meta-data requirements will be shared. We also aim to create an open forum for international researchers to share expertise in single-cell laboratory methodology for challenging (low-cellularity, matrix-rich) musculoskeletal tissues, as well as generating a community resource of computational workflows and encouraging benchmarking of single-cell methodologies and cellular annotations to identify high-quality but economical techniques. The expertise, data and methodologies developed will inform the final roadmap, delivering breadth and depth to the musculoskeletal atlas.

The musculoskeletal HCA network is an open community that will foster collaboration and shared expertise. Researchers of any discipline, at any career stage, working or initiating work in the transcriptomic, epigenomic and proteomic analysis of musculoskeletal tissues at the single-cell level are encouraged to join the growing community within the musculoskeletal network. Although our current focus is on human tissue, we welcome the chance to translate the expertise and insight from our colleagues working with animal models or in vitro systems. We also aim to hold quarterly virtual meetings to disseminate research on HCA-related projects and to serve as a platform to generate new ideas, build new collaborations, develop the musculoskeletal atlas roadmap and identify careerdevelopment opportunities that will accelerate the field of musculoskeletal research. 
A cohesive and complementary approach from a critical mass of international researchers will be vital to progress, and teams are encouraged to upload relevant data to the HCA Data Coordination Platform. Underpinning these ambitions is our network's core belief that cellular atlases must be viewed as a fundamental resource for the public good. We have a moral and ethical duty to ensure that all methods, code and data generated are ethnically diverse and openly available for the wider scientific communities. It is exciting to look forward and see how an community-driven effort would expedite delivery of robust and representative cellular maps of the musculoskeletal system, paving the way for improved understanding of disease mechanism and therapeutic development.

1. Paper, W. THE HUMAN CELL ATLAS. https://www.humancellatlas.org/wpcontent/uploads/2019/11/HCA_WhitePaper_180ct2017-copyright.pdf (2017).

2. Cieza, A. et al. Global estimates of the need for rehabilitation based on the Global Burden of Disease study 2019: a systematic analysis for the Global Burden of Disease Study 2019. Lancet (London, England) 396, 2006-2017 (2021).

3. McMahon, S. B., Dargan, P., Lanas, A. \& Wiffen, P. The burden of musculoskeletal pain and the role of topical non-steroidal anti-inflammatory drugs (NSAIDs) in its treatment. Ten underpinning statements from a global pain faculty. Curr. Med. Res. Opin. 1-11 (2020) doi:10.1080/03007995.2020.1847718.

4. GBD 2017 Disease and Injury Incidence and Prevalence Collaborators, S. L. et al. Global, regional, and national incidence, prevalence, and years lived with disability for 354 diseases and injuries for 195 countries and territories, 1990-2017: a systematic analysis for the Global Burden of Disease Study 2017. Lancet (London, England) 392, 1789-1858 (2018).

5. Lee, R. C. et al. Total-body skeletal muscle mass: development and cross-validation of anthropometric prediction models. Am. J. Clin. Nutr. 72, 796-803 (2000).

6. Tan, A., Strauss, V. Y., Protheroe, J. \& Dunn, K. M. Epidemiology of paediatric presentations with musculoskeletal problems in primary care. BMC Musculoskelet. Disord. 19, 40 (2018).

7. Vieira Braga, F. A. et al. A cellular census of human lungs identifies novel cell states in health and in asthma. Nat. Med. 25, 1153-1163 (2019).

8. Aizarani, N. et al. A human liver cell atlas reveals heterogeneity and epithelial progenitors. Nature 572, 199-204 (2019).

\section{Competing interests}

The authors declare no competing interests. 


\section{Related links}

Human Cell Atlas: https://www.humancellatlas.org/

Musculoskeletal network: https://www.humancellatlas.org/areas-of-impact/

HCA Data Coordination Platform: https://data.humancellatlas.org/contribute

Figure 1

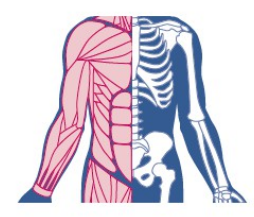

Global systematic collection

of musculoskeletal tissues

from across anatomy
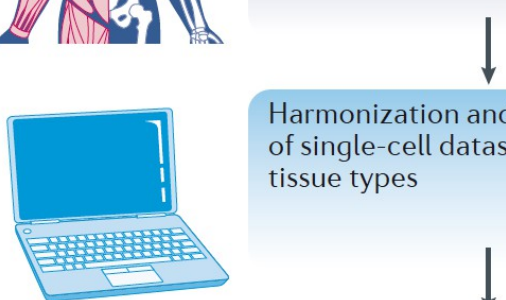

Harmonization and annotation

of single-cell datasets across

tissue types

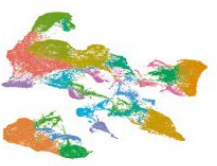

Comprehensive development

of multidimensional maps of

human musculoskeletal tissues

\section{Key steps in delivery of a single cell atlas of the Musculoskeletal system.}

A robust and representative single cell resolution atlas of the musculoskeletal system requires community effort to ensure systematic tissue collection and harmonised data analysis across all musculoskeletal tissues. . 